\title{
Estimulación eléctrica neuromuscular en el paciente crítico. Una revisión narrativa
}

\section{Neuromuscular Electrical Stimulation in the Critically III Patient. A narrative review}

Ruvistay Gutiérrez-Arias, Mg. ${ }^{1,2, *}$, Yorschua Jalil Contreras, Mg. ${ }^{2,3,4}$

1 Unidad de Kinesiología, Unidad de Paciente Crítico, Instituto Nacional del Tórax. Santiago, Chile.

2 Exercise and Rehabilitation Sciences Laboratory, School of Physical Therapy, Faculty of Rehabilitation Sciences, Universidad Andres Bello, Santiago 7591538, Chile.

3 Departamento Ciencias de la Salud, Carrera de Kinesiología, Facultad de Medicina, Pontificia Universidad Católica de Chile. Santiago, Chile.

4 Programa de Doctorado Ciencias Médicas, Pontificia Universidad Católica de Chile, Escuela de Medicina. Santiago, Chile.

Financiamiento: No se contó con fuentes de financiamiento externo.

Conflicto de interés: Los autores declaran no tener conflictos de interés.

Fecha de recepción: 12 de mayo de 2021 / Fecha de aceptación: 18 de junio 2021

\begin{abstract}
Advances in the treatment of critically ill patients have led to increased survival rates. However, survivors often experience significant consequences associated with the conventional model of care, in which patients tend to remain inactive, particularly during treatment with mechanical ventilation (MV) and prolonged use of sedation. This can result in mental, psychological, speech and swallowing disorders, and/or physical sequelae, including muscle weakness and consequent physical disability, which in many cases persist after discharge. Current evidence suggests that neuromuscular electrical stimulation has a positive effect on the mass and strength of the stimulated muscles, and may also induce systemic pro-myogenic, anti-inflammatory and regenerative effects, which could influence a decrease in MV time among other benefits. These effects could impact outcomes relevant to decision-makers, such as reduced intensive care unit length of stay and total hospitalization time.
\end{abstract}

Key words: Electric stimulation therapy, intensive care units, muscle weakness.

\section{RESUMEN}

Los avances en el tratamiento de pacientes críticamente enfermos han llevado a una mayor tasa de supervivencia. Sin embargo, los sobrevivientes a menudo experimentan consecuencias significativas asociadas al modelo convencional de atención, en el cual los pacientes tienden a permanecer inactivos, particularmente durante el tratamiento con ventilación mecánica (VM) y uso prolongado de sedación. Esto puede determinar trastornos mentales, psicológicos, del habla y deglución, y/o secuelas físicas, entre las que destacan la debilidad muscular y la consecuente discapacidad física, la que en muchos casos persisten tras el alta. La evidencia actual sugiere que la estimulación eléctrica neuromuscular tiene un efecto positivo sobre la masa y fuerza de los músculos estimulados, pudiendo además inducir efectos sistémicos pro-miogénicos, antiinflamatorios y regenerativos, que podrían influir en una disminución del tiempo de VM entre otros beneficios. Estos efectos podrían impactar desenlaces relevantes para los tomadores de decisiones, como la disminución de la estadía en unidades de cuidados intensivos y el tiempo de hospitalización total.

Palabras clave: Estimulación eléctrica, unidades de cuidados intensivos, debilidad muscular.

rgutierrez@torax.cl

ruvistay.gutierrez@gmail.com

https://orcid.org/0000-0003-1881-9316 


\section{Introducción}

M illones de personas son ingresadas anualmente a unidades de cuidados intensivos (UCI) en todo el mundo, reportándose la admisión de 1 cada 200 adultos cada año[1]. La demanda de camas críticas ha ido aumentando gradualmente y se espera que esta tendencia siga debido a la mayor esperanza de vida, enfermedades crónicas asociadas a estilos de vida poco saludables, y a la aparición de nuevas enfermedades[2]. Chile casi doblo el número de camas críticas entre 2002 y 2012, pasando de 773 a 1.270 camas[3], sin embargo, actualmente alcanza su máximo histórico respecto a su dotación y ocupación.

Los avances en el tratamiento de pacientes críticamente enfermos han llevado a una mayor tasa de supervivencia. Sin embargo, los sobrevivientes frecuentemente experimentan consecuencias significativas asociadas al modelo convencional de atención, en el cual los pacientes tienden a permanecer inactivos, particularmente durante el tratamiento con ventilación mecánica (VM) y uso prolongado de sedación[4]. Esto puede determinar trastornos mentales, psicológicos, del habla y deglución, y/o secuelas físicas, entre las que destacan la debilidad muscular y la consecuente discapacidad física, la que en muchos casos persiste tras el alta[4],[5]. Es por esto que el acceso oportuno a rehabilitación, y los recursos necesarios para ello, aparecen como un desafío sanitario relevante, siendo necesario un cambio de paradigma hacia la prevención en etapas precoces de la enfermedad critica[6], en donde la estimulación eléctrica neuromuscular (EENM) puede jugar un rol complementario en el contexto de desgaste muscular acelerado en pacientes en estado crítico independiente del trastorno inicial, dado el origen fisiopatológico común que los afecta, siendo el shock séptico el más frecuentemente referido en esta revisión.

\section{Debilidad muscular en UCl en el paciente crítico}

La debilidad adquirida en UCI (DA-UCI) constituye uno de los trastornos más prevalentes y característicos en los pacientes admitidos en $\mathrm{UCl}$, afectando entre el $30 \%$ y $90 \%$ de esta población[7],[8]. Se caracteriza por un acelerado desgaste muscular que afecta tanto a músculos periféricos como respiratorios, y ha sido asociada con peores desenlaces a corto y largo plazo, incluyendo el uso prolongado de VM, mayor estadía en $\mathrm{UCl}$, disminución en la función pulmonar a 5 años pos alta hospitalaria y aumento de la mortalidad[9].

Los pacientes pueden experimentar una pérdida del grosor muscular diafragmático del orden del $20 \%$ a los 3 días de VM y una diminución en el grosor muscular de cuádriceps de un $10 \%$ luego de una semana[10], siendo fenómenos relacionados, pero distintos en relación a sus cursos temporales de aparición, destacando la temprana afectación de la musculatura respiratoria, la cual ha sido reportada como un factor independiente para mortalidad y uso prolongado de VM[11].

En términos generales, los factores de riesgo para el desarrollo de desgaste muscular son múltiples e incluyen sedación profunda, sepsis, hiperglucemia, falla multiorgánica, uso de corticosteroides, uso de bloqueo neuromuscular y VM, estando este último íntimamente ligado a la inactividad diafragmática y su consecuente disfunción. Además, la inflamación aguda en etapas precoces, la que podría verse potenciada por una in- adecuada terapia nutricional[12], es un poderoso potenciador del catabolismo muscular y eventual proteólisis, posiblemente debido al aumento de la producción de citoquinas pro inflamatorias, autofagia y empeoramiento del estrés oxidativo[13].

\section{Prevención de la debilidad muscular en UCI}

Actualmente, la limitación y uso racional del bloqueo neuromuscular de manera continua[14], y la movilización y activación muscular temprana de los pacientes en UCI ha sido establecida como una estrategia capaz de reducir el desacondicionamiento físico y mitigar la disfunción muscular apendicular[15]. Interesantemente, esta contracción muscular tendría un impacto positivo no sólo debido a sus efectos locales sobre el tejido muscular activado y el consecuente aumento del trofismo muscular, reducción de la actividad proteolítica y resultante preservación de la fuerza muscular, sino también por sus potenciales efectos sistémicos dirigidos a vías anabólicas en todo el cuerpo, actuando como un órgano endocrino a través de la secreción de mioquinas[16].

Las mioquinas, entre las que encontramos a la Miostatina, factor neurotrófico derivado del cerebro (Brain derived neurotrophic factor; BDNF), IL-15, Decorina, Mionectina e IL-6, entre otras, corresponden a una amplia gama de citoquinas y quimioquinas capaces de mediar activamente interacciones célulacélula y órgano-órgano y, por lo tanto, podrían promover un perfil antiinflamatorio y de regeneración muscular a distancia en otros grupos musculares, así como también modular la función y metabolismo de otros órganos (ej.: hígado y cerebro) [16],[17]. Desafortunadamente, la contracción muscular activa se ve limitada en las primeras etapas de la atención en $\mathrm{UCl}$, donde la sedación y uso de VM son vitales, generando un círculo vicioso que retrasara el inicio de la actividad muscular[18]. En este contexto, la EENM, surge como alternativa al ejercicio activo en pacientes no cooperadores, la cual podría desencadenar todos los efectos de una activación muscular tanto a nivel local como a distancia[19].

\section{Estimulación eléctrica neuromuscular y sus efectos locales en el paciente crítico}

Definido como la aplicación de una serie de estímulos intermitentes a la musculatura esquelética superficial, con el objetivo principal de desencadenar contracciones musculares visibles debido a la activación de las ramas nerviosas intramusculares, el concepto de EENM no es nuevo[20]. La evidencia indica que su uso como modalidad de entrenamiento permite la preservación y recuperación de la masa y función muscular en algunos pacientes, y mejoras en la fuerza muscular en sujetos sanos y deportistas[21].

Sin embargo, la gran mayoría de los estudios acerca de la EENM como método de entrenamiento se han centrado en músculos sanos más que en músculos dañados, por lo que su potencial permanece en parte inexplorado, especialmente con respecto a sus posibles efectos sistémicos en contextos patológicos como la DA-UCI[22]. Varios autores han dado recomendaciones razonables para la implementación de la EENM en pacientes críticamente enfermos, con el fin de maximizar su rendimiento físico en términos de fuerza y preservación de la masa muscular en extremidades[21],[22]. 
Gerovasili et al., utilizando ecografía, demostraron en 26 pacientes críticamente enfermos con VM, que la EENM parece preservar el diámetro del músculo transversal del vasto intermedio y recto femoral del cuádriceps, siendo una intervención segura y potencialmente útil para prevenir el desgaste muscular[25]. Rodríguez et al., quienes aplicaron EENM sólo en un lado del cuerpo de pacientes sépticos con VM, encontraron un puntaje significativamente mayor en la escala manual de fuerza muscular de la Medical Research Council (MRC) en el lado estimulado, en comparación con el hemicuerpo control[26]. Similarmente, Dirks et al., usando biopsias de cuádriceps de pacientes completamente sedados que recibieron EENM también en un solo lado, observaron un menor grado de atrofia muscular en la extremidad inferior estimulada [27].

\section{Estimulación eléctrica neuromuscular y sus efectos sistémicos en el paciente crítico}

Routsi et al., mostraron que la EENM aplicada sobre el cuádriceps de pacientes con VM podría no sólo prevenir la DA-UCI, sino que interesantemente sería capaz de disminuir de manera significativa el tiempo en $\mathrm{VM}(\mathrm{P}=0,003)$ del grupo que recibió EENM (mediana de 1 día; rango 0 a 10 días) en comparación al grupo control (mediana de 3 días; rango 0 a 44 días), sugiriendo una relación entre la EENM periférica y el rendimiento de los músculos respiratorios[28]. Abu-Khaber et al., reportaron resultados similares, sugiriendo que la EENM no sólo tendría un impacto positivo a nivel local, es decir donde se aplica, sino que tendría un impacto sistémico[29]. Esto podría darse posiblemente a la liberación de mioquinas desde el músculo, las que determinarían un perfil protector (pro-miogénico, antiinflamatorio y regenerativo) capaz de facilitar el proceso de liberación de la VM[29]

La relación entre EENM y la secreción de mioquinas ha sido mostrada previamente en pacientes con insuficiencia cardíaca. Karavidas et al., evaluaron el impacto de la EENM sobre marcadores sanguíneos periféricos de la activación inmune y la inflamación en 24 pacientes con insuficiencia cardíaca moderada a severa sometidos a un programa de entrenamiento de 6 semanas basado en EENM, informando una reducción significativa de TNF- $\alpha(-11,5 \pm 8,9 \%)$ y un aumento en la relación IL-10/ TNF- $\alpha(37,1 \pm 29,4 \%)$. Esto sugiere un efecto antiinflamatorio[30]. Sin embargo, vale destacar que la modalidad de EENM aplicada difiere de la clásicamente entendida como EENM, siendo una versión que incorpora la activación voluntaria como un complemento sinérgico a la contracción provocada por el estímulo eléctrico, estrategia conocida como estimulación eléctrica funcional o FES; modalidad que escapa de los objetivos de esta revisión en el contexto de pacientes en etapas tempranas de hospitalización no colaboradores. De manera similar, Dobšák et al., mostraron que la EENM reduce los niveles de proteína $C$ reactiva (PCR) alrededor del $62 \%$ en pacientes con insuficiencia cardíaca[31].

La EENM puede modificar el entorno de citoquinas proinflamatorias y promover la secreción de mioquinas como el BDNF relacionado con la regeneración muscular, y que a nivel sérico aumentaría significativamente tras el uso de EENM, evidenciándose un cambio incluso mayor al obtenido tras ejercicio voluntario en hombres sanos[32].

Treinta minutos de EENM también serían capaces de inducir cambios en el perfil de citoquinas en sangre periférica de sujetos sanos, similares a los efectos alcanzados con ejercicio activo, mostrando un aumento significativo $(p=0,001)$ del valor máximo de IL-6 de 0,65 pg/ml en comparación con su valor medio pre-EENM de 1,04 pg/ml, y una disminución significativa en el valor medio de IL-1 de 0,08 pg/ml a 0,02 pg/ml $(p=0,041)$ y TNF- $\alpha$ de $2,42 \mathrm{pg} / \mathrm{ml}$ a $2,16 \mathrm{pg} / \mathrm{ml}(p=0,021)$ [33]. Estos cambios en las citoquinas circulantes podrían mediar los potenciales efectos sistémicos de la EENM en la prevención del desgaste muscular, mostrando también una tendencia a la disminución de los marcadores de daño muscular, como el lactato y la creatina quinasa[33].

\section{Desenlaces clínicos reportados: Revisiones sistemáticas y metaanálisis}

Revisiones sistemáticas (RS) actualmente publicadas han reportado diferentes resultados, incluso contradictorios (Tabla 1), para una diversidad de desenlaces clínicos, lo que quizás ha jugado un rol en la pobre masificación de esta intervención. Estas diferentes conclusiones entre las revisiones sistemáticas pueden deberse, obviamente, a la continua publicación de nuevos ensayos clínicos (ECAs), pero también a criterios de elegibilidad levemente distintos, la no inclusión de estudios que debieron ser identificados por la estrategia de búsqueda de cada revisión, y al análisis diferencial en cuanto al riesgo de sesgo de los estudios primarios, lo que puede llevar a una estimación diferente de la efectividad de la EENM en los diferentes desenlaces. Estos desenlaces van desde efectos locales, como la fuerza de la musculatura apendicular, especialmente del cuádriceps, hasta resultados indirectos como la duración de la estadía en UCI (Figura 1).

En cuanto a los efectos locales, Maffiuletti et al., en su RS publicada en el año 2013, reportaron de manera cualitativa que la EENM, sumada al cuidado habitual, mejora la fuerza muscular, con ningún o poco efecto en la masa muscular[34]. Sin embargo, Parry et al., en su RS publicada el mismo año, reportaron que la EENM preserva tanto la masa como la fuerza muscular[35, misma conclusión a la que llegaron Wageck et al.[23], y Trethewey et al. [24] en el año 2014 y 2019, respectivamente. Parry et al., también destacaron que los efectos de la EENM son más claros en los pacientes de menor gravedad y con una estadía hospitalaria más prolongada[35]. Además, el metaanálisis (MA) reportado en la RS de Burke et al., publicado el año 2014, y que consideró tres ECAs, mostró que la EENM logró 0,93 puntos más (IC 95\% 0,51 a 1,35) en la escala de la MRC en comparación al grupo control[36], resultados similares a los publicados por Liu et al., quien en su MA reportado el año 2020 que incluyó cinco ECAs, mostró que los pacientes sometidos a EENM tienen un puntaje en la escala MRC de 1,78 puntos más (IC 95\% 0,44 a 3,12) en comparación con el cuidado habitual[37]. Sin embargo, Zayed et al., reportaron en el año 2019 que no existió diferencia en la fuerza muscular al alta de la UCl entre el grupo que recibió EENM y el grupo control con una diferencia de medias (DM) de 0,45 puntos (IC 95\% -2,89 a 3,80)[19].

En cuanto a los efectos sistémicos, si bien la RS de Zayed et al., no mostró diferencia en la duración de VM al comparar el uso de EENM y el cuidado estándar (DM = -2,07 días (IC $95 \%-5,06$ a 0,92); 4 ECAs [19], el MA de Liu et al., reportó 
Tabla 1. Revisiones sistemáticas sobre la efectividad de la estimulación eléctrica neuromuscular en pacientes críticos

\section{$\mathrm{N}^{\circ}$ de ECAs (N-pacientes)}

\begin{tabular}{ll}
\hline Autor y año & Título \\
Mafiulletti 2013 & $\begin{array}{l}\text { Neuromuscular electrical } \\
\text { stimulation for preventing } \\
\text { skeletal-musde weakness and } \\
\text { wasting in critically ill patients: } \\
\text { a systematic review }\end{array}$
\end{tabular}

Parry 2013

Electrical Muscle Stimulation in the Intensive Care Setting: A Systematic Review
8 ECAs

(172 pacientes)

9 ECAs

(136 pacientes)

Burke 2014

An evaluation of neuromuscular electrical stimulation in critical care using the ICF framework: a systematic review and metaanalysis

Wageck 2014 Application and effects of neuromuscular electrical stimulation in critically ill patients: Systematic review

Trethewey 2019 Interventions for the management and prevention of sarcopenia in the critically ill: A systematic review

Zayed 2019

Liu 2020

Effects of neuromuscular electrical stimulation in critically ill patients: A systematic review and meta-analysis of randomised controlled trils

Intervention effect of neuromuscular electrical stimulation on ICU acquired weakness: a meta-analysis
11 ECAs

8 ECAs (252 pacientes)

11 ECAs (549 pacientes)

6 ECAs (718 pacientes)

11 ECAs (576 pacientes)

12 ECAs (530 pacientes) (432 pacientes)

\section{Conclusiones}

La EENM añadida a la atención habitual demostró ser más eficaz que el cuidado habitual en prevenir la debilidad músculo esquelética en pacientes críticos. Sin embargo, no hay pruebas concluyentes de su beneficio en la prevención de la atrofia muscular

La EENM es una intervención prometedora; sin embargo, la evidencia es contradictoria sobre su eficacia cuando se administrada de forma aguda

La EENM, como complemento a las prácticas actuales de rehabilitación en pacientes críticos, puede mantener la fuerza muscular

La EENM tiene buenos resultados cuando se utiliza para el mantenimiento de la masa y la fuerza muscular en pacientes críticos

La EENM puede preservar la masa y la función muscular en pacientes con enfermedad critica

El EENM combinado con la atención habitual no se asoció con diferencias significativas en la fuerza muscular global, mortalidad en $\mathrm{UCl}$, duración de la VM o la duración de la estancia en UCI

La aplicación temprana de la EENM en pacientes críticos puede prevenir la DA-UCI y mejorar su calidad de vida al mejorar su fuerza muscular, acortar la duración de la VM, la duración de la estancia en UCI y la duración total de la estancia en el hospital

La EENM puede reducir ligeramente la duración de la VM invasiva en comparación a la atención habitual o placebo

\section{Calidad de la evidencia}

La puntuación media (DE) de la escala PEDro de los ECAs incluidos fue de $5,5(1,5)$ con puntuaciones que iban de 4 a 8 (es decir, de calidad moderada a alta)

La calidad general según la escala PEDro de los ECAs incluidos se calificó de "regular" a "buena", y seis ECAs obtuvieron una puntuación entre 4 y 8

Según la escala RoB de Cochrane, el riesgo de sesgo fue alto en ocho ECAs, con un estudio calificado como "poco claro"

Aunque dos estudios fueron calificados con una calidad alta, la mayoría de los estudios tenían calidad de intermedia a baja (escala PEDro $f 5$ puntos)

La mayoría de los ECAs estuvieron sujetos a un alto riesgo de sesgo (según escala RoB de Cochrane)

El riesgo de sesgo fue de moderado a alto (según escala RoB de Cochrane)

La mayoría de los ECAs estuvieron sujetos a un alto riesgo de sesgo (según escala RoB de Cochrane)

La mayoría de los ECAs estuvieron sujetos a un alto riesgo de sesgo (según escala RoB de Cochrane)

ECAs: Ensayos Clínicos Aleatorizados; EENM: Estimulación Eléctrica Neuromuscular; DE: Desviación Estándar; RoB: Risk of Bias; UCl: Unidad de Cuidados Intensivos; VM: Ventilación Mecánica; DA-UCI: Debilidad Adquirida en UCI.

un menor tiempo de VM en los pacientes sometidos a EENM, con una diferencia de medias estandarizada (DME) de -0,65 DE ((IC 95\% -1,03 a -0,27); 6 ECAs)[37], hallazgo confirmado por la recientemente publicada RS de Gutiérrez-Arias et al., la cual incluyó 10 ECAs en su MA, y la que reportó que la EENM disminuye en 2,68 días (IC 95\% -4,35 a -1,02) la duración de la VM en comparación al cuidado habitual o EENM simulada, lo que podría ser levemente más marcado en pacientes con EPOC como patología de base (DM -2,90 días (IC 95\% -4,58 a -1,23); 3 ECAs)[38].

Finalmente, en relación con desenlaces indirectos posiblemente impactados por la aplicación de la EENM, en algunos casos los reportes también son contradictorios. La RS de

Zayed et al., no mostró diferencias en el tiempo de estadía 


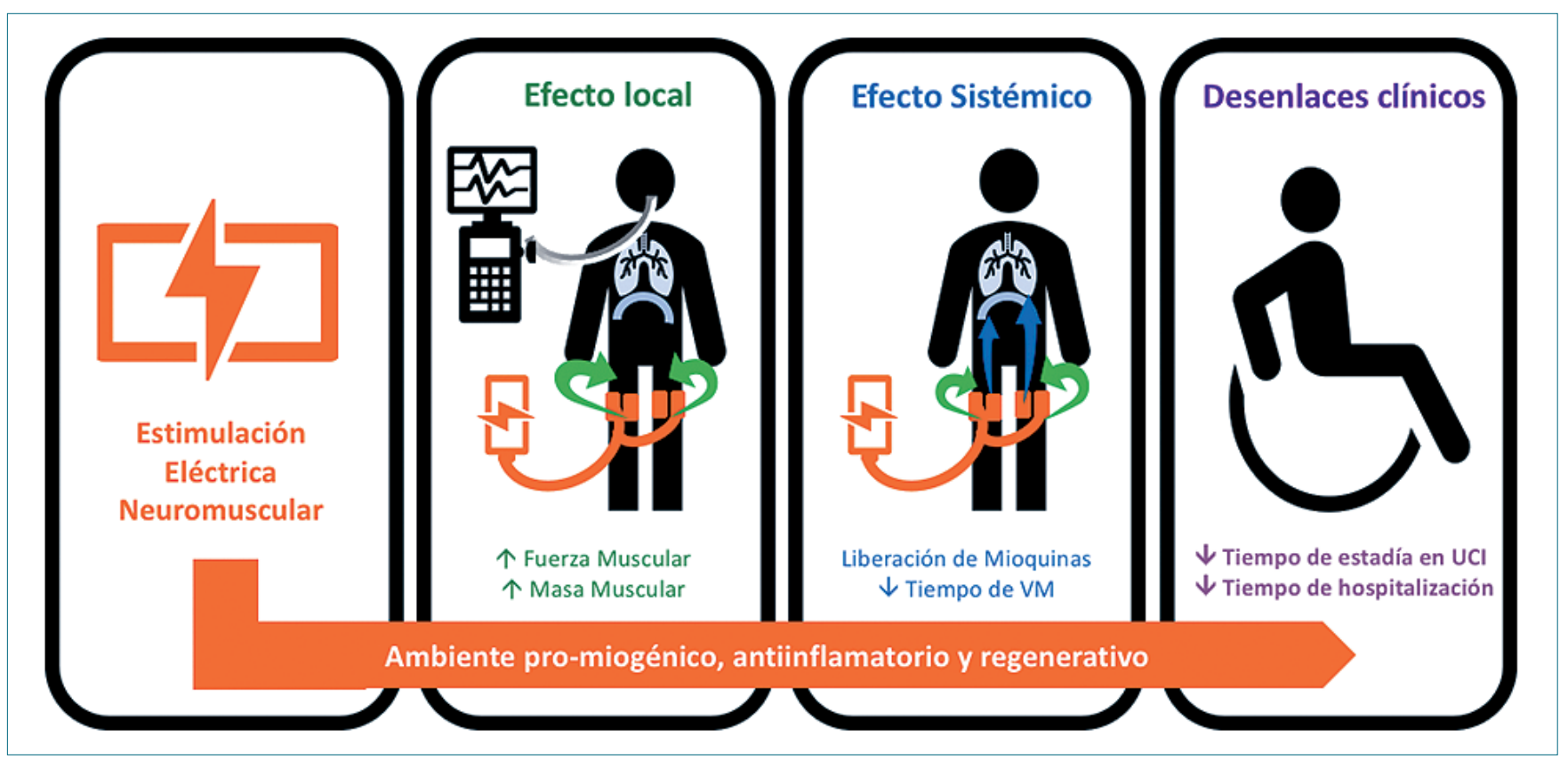

Figura 1. Efectos locales, sistémicos y potencial impacto clínico de la estimulación eléctrica neuromuscular.

en UCI (DM -3,06 días (IC 95\% -9,79 a 3,68); 3 ECAs)[19]. Por el contrario, Liu et al., reportó tanto una menor duración de estadía en UCI (DM -3,41 días (IC 95\% -4,58 a -2,24); 4 ECAs) como en el total de la hospitalización (DM -3,97 días (IC 95\% $-6,89$ a $-1,06) ; 3$ ECAs)[37]. Además, tanto Zayed et al., (RR 1,30 (IC 95\% 0,95 a 1,78); 4 ECAs)[19] como Liu et al., (RR 1,07 (IC 95\% 0,62 a 1,84); 3 ECAs)[37] coinciden en que no existe diferencia significativa en la mortalidad entre los grupos que fueron sometidos a EENM y el cuidado habitual.

\section{Dosificación de la estimulación eléctrica neuromuscular}

Los estudios que han buscado determinar la efectividad de la EENM, como método de prevención y tratamiento de las diferentes disfunciones musculares del paciente crítico, han aplicado esta estrategia con algunas diferencias en cuanto a su localización y dosificación. Lo electrodos, los cuales pueden tener tamaños entre $5 \times 5$ a $5 \times 10 \mathrm{~cm}$ con la finalidad de producir un adecuado reclutamiento de la unidad motora[39], frecuente son instalados en los músculos asociados con la deambulación[26],[27],[37], debido probablemente a que la EENM tiene como último fin favorecer la recuperación de la independencia funcional de los pacientes críticos. La función del cuádriceps está asociada directamente con diferentes desenlaces clínicamente relevantes, y que en términos de generación de mioquinas es deseable la activación de la mayor cantidad de masa muscular disponible, siendo el cuádriceps uno de los más potentes en este sentido[41]. Sin embargo, estudios específicos han reportado también la aplicación de EENM en músculos del complejo toraco-abdominal[39],[40],[41],[42], los que buscaban evaluar si la aplicación directa en la musculatura relacionada con la respiración tenía impacto en la liberación de la VM[46] (Tabla 2).

Habitualmente, los programas de EENM han sido diseñados para realizarse una a dos veces al día, y se ha reportado una duración total entre 10 y 14 días[35],[36]. Sin embargo, en la práctica clínica es habitual que la aplicación de dicha intervención se prolongue durante toda la estadía en unidades de paciente crítico, teniendo en cuenta que incluso podría favorecer la desvinculación del soporte ventilatorio en pacientes con VM prolongada. En cuanto a la duración del pulso y la frecuencia

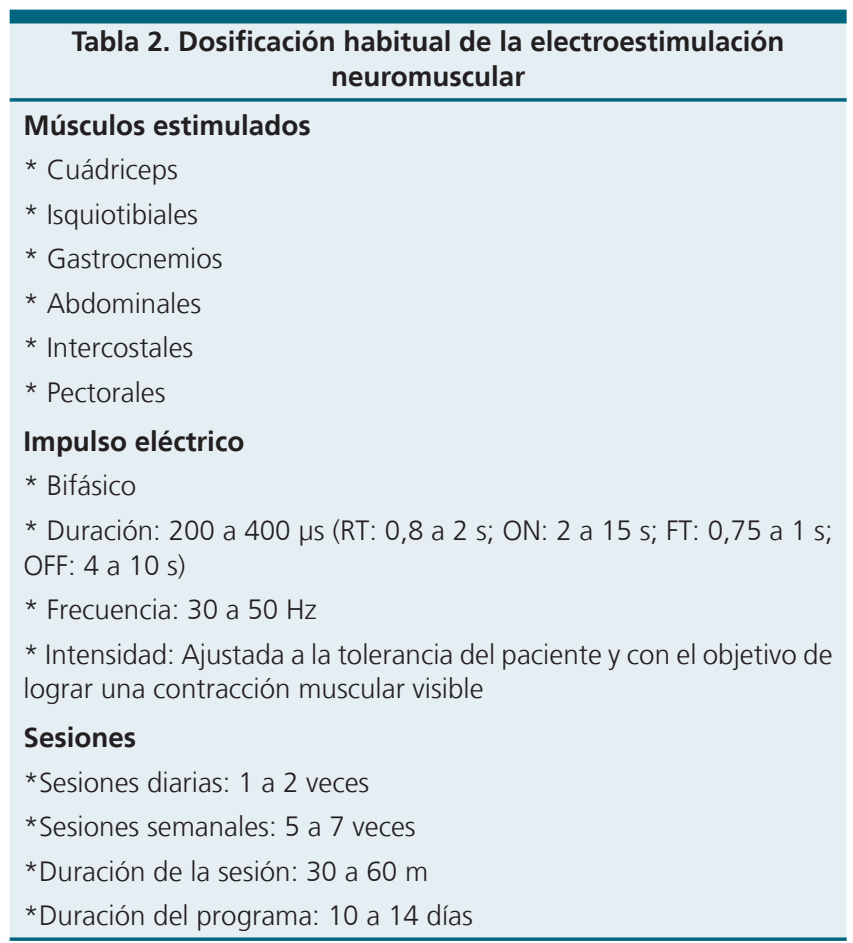

$\mu$ s: Microsegundo; s: segundis; m: minutos; Hz: Hertz; RT: Rise time; FT: Fall time. 
del estímulo, se han reportado diferentes protocolos de programación, oscilando habitualmente entre 200 y $400 \mu$ s, y de 30 a $50 \mathrm{~Hz}[35],[36]$, respectivamente (Tabla 2).

Si bien la EENM ha demostrado ser una intervención segura en pacientes con enfermedad crítica, reportándose sólo infrecuentes molestias locales, como enrojecimiento y prurito, y ningún evento adverso mayor[47] dicha intervención debe ser aplicada por profesionales entrenados y teniendo en cuenta las contraindicaciones para su uso. De las cuales se destacan la aplicación sobre zonas de la piel con lesiones infecciosas, cortantes o quemaduras, fracturas subyacentes, trombosis venosa profunda sin tratamiento de anticoagulación. Además, se debe descartar su uso en enfermedades sistémicas como lupus eritematosos sistémico, rabdomiólisis, enfermedades cardiacas con arritmias asociadas o con necesidad de marcapasos, alteración de la coagulación y otras enfermedades, sistémicas o locales, que provoquen disminución de la sensibilidad en la zona que se quiera estimular[48],[49].

\section{Futuros desafíos en la investigación}

Si bien la aplicación de la EENM en esta población es considerada como una intervención prometedora y que debiera ser incluida como una técnica estándar dentro los programas de prevención y tratamiento de las disfunciones físicas de los pacientes críticamente enfermos, son necesarios estudios fisiológicos acerca de su efecto en la etapa precoz de la enfermedad crítica, donde mayor desgaste muscular se produce, apuntando a identificar las interacciones célula-célula y órgano-órgano (por ejemplo, mioquinas a nivel local y sistémico) capaces de condicionar un perfil muscular protector, previniendo las manifestaciones clínicas severas derivadas de la estancia en $\mathrm{UCI}$. Además, deben desarrollarse en complemento estudios clínicos con un adecuado tamaño muestral y un diseño metodológico que considere grupos controles con intervenciones que no confundan el efecto real de la EENM. Igualmente, se deberían conducir más ECAs que comparen las distintas dosificaciones de la EENM, en cuanto a la duración de las sesiones, la frecuencia de aplicación de la terapia, y las diferentes programaciones de los dispositivos, con la finalidad de determinar la mejor forma de entregar esta intervención.

En cuanto a los estudios enfocados a la síntesis de evidencia, se hace necesario una evaluación inicial de las RS y MA publicados, debido a la discrepancia en sus resultados, considerando que incluso incluyen estudios primarios similares. Además, futuras RS deben considerar la heterogeneidad clínica en primera instancia, y no solo basarse en criterios estadísticos para la realización de MA, con la finalidad de identificar las características de las poblaciones que son susceptibles de beneficiarse en mayor medida de la EENM, y esclarecer la dosificación con mayor potencial de esta intervención.

\section{Conclusión}

La evidencia actual sugiere que la EENM podría tener un efecto positivo sobre la masa y fuerza de los músculos estimulados, pudiendo tener efectos sistémicos pro-miogénicos, antiinflamatorios y regenerativos que podrían influir en una disminución del tiempo de VM. Estos efectos podrían impactar en desenlaces relevantes para los tomadores de decisiones, como la disminución de la estadía en UCI y el tiempo de hospitalización total. Sin embargo, la calidad de la evidencia que sustenta su efectividad tiene un riesgo de sesgo moderado a alto. Por lo que más y mejores estudios, en términos de diseño metodológi$\mathrm{co}$, son necesarios para discriminar su real efectividad y potencial aporte en el cuidado de esta vulnerable población, y así la EENM pueda ser considerada como una potencial herramienta en la prevención y rehabilitación de las diferentes disfunciones neuromusculares que padecen los pacientes críticos.

Agradecimientos: Yorschua Jalil Contreras agradece el soporte parcial de CONICYT-PFCHA/Doctorado Nacional 2019-Folio 21191025.

\section{Referencias}

1. Garland A, Olafson K, Ramsey CD, Yogendran M, Fransoo R. Epidemiology of critically ill patients in intensive care units: a population-based observational study. Crit Care. 2013 Sep;17(5):R212. https://doi.org/10.1186/cc13026 PMID:24079640

2. Sen-Crowe B, Sutherland M, McKenney M, Elkbuli A. A Closer Look Into Global Hospital Beds Capacity and Resource Shortages During the COVID-19 Pandemic. J Surg Res. 2021 Apr;260:5663. https://doi.org/10.1016/j.jss.2020.11.062 PMID:33321393

3. Gálvez GS, González DH, Labarca ME, et al. Medicina intensiva en Chile: desafíos para su desarrollo. Documento de la Comisión Nacional de Medicina Intensiva del Ministerio de Salud. Rev Med Chil. 2013;141(1):90-4. https://doi.org/10.4067/S003498872013000100012.

4. Puthucheary ZA, Rawal J, McPhail M, Connolly B, Ratnayake G, Chan $P$, et al. Acute skeletal muscle wasting in critical illness. JAMA. 2013 Oct;310(15):1591-600. https://doi.org/10.1001/ jama.2013.278481 PMID:24108501

5. Iwashyna TJ, Ely EW, Smith DM, Langa KM. Long-term cognitive impairment and functional disability among survivors of severe sepsis. JAMA. 2010 Oct;304(16):1787-94. https://doi. org/10.1001/jama.2010.1553 PMID:20978258

6. Ahmed H, Patel K, Greenwood DC, Halpin S, Lewthwaite P, Salawu $A$, et al. Long-term clinical outcomes in survivors of severe acute respiratory syndrome and Middle East respiratory syndrome coronavirus outbreaks after hospitalisation or ICU admission: A systematic review and meta-analysis. J Rehabil Med. 2020 May;52(5):jrm00063. https://doi.org/10.2340/16501977-2694 PMID:32449782

7. Kress JP, Hall JB. ICU-acquired weakness and recovery from critical illness. N Engl J Med. 2014 Apr;370(17):1626-35. https://doi. org/10.1056/NEJMra1209390 PMID:24758618

8. Mehta SR, Granger CB, Boden WE, Steg PG, Bassand JP, Faxon $D P$, et al.; TIMACS Investigators. Early versus delayed invasive intervention in acute coronary syndromes. N Engl J Med. 2009 May;360(21):2165-75. https://doi.org/10.1056/NEJMoa0807986 PMID:19458363

9. Garnacho-Montero J, Madrazo-Osuna J, García-Garmendia JL, Ortiz-Leyba C, Jiménez-Jiménez FJ, Barrero-Almodóvar A, et al. Critical illness polyneuropathy: risk factors and clinical consequences. A cohort study in septic patients. Intensive Care Med. 2001 Aug;27(8):1288-96. https://doi.org/10.1007/ s001340101009 PMID:11511941 
10. Gruther W, Benesch T, Zorn C, Paternostro-Sluga T, Quittan $M$, Fialka-Moser $\mathrm{V}$, et al. Muscle wasting in intensive care patients: ultrasound observation of the $\mathrm{M}$. quadriceps femoris muscle layer. J Rehabil Med. 2008 Mar;40(3):185-9. https://doi. org/10.2340/16501977-0139 PMID:18292919

11. Jung B, Moury PH, Mahul M, de Jong A, Galia F, Prades A, et al. Diaphragmatic dysfunction in patients with ICU-acquired weakness and its impact on extubation failure. Intensive Care Med. 2016 May;42(5):853-61. https://doi.org/10.1007/s00134-0154125-2 PMID:26572511

12. Liang K, Puthucheary Z. Association of nutritional delivery on skeletal muscle wasting and inflammation in critically ill adult patients: a systematic review. Proc Nutr Soc. 2021;80 OCE1:E27. https://doi.org/10.1017/S0029665121000288.

13. Dres M, Goligher EC, Heunks LM, Brochard LJ. Critical illnessassociated diaphragm weakness. Intensive Care Med. 2017 Oct;43(10):1441-52. https://doi.org/10.1007/s00134-017-49284 PMID:28917004

14. Renew JR, Ratzlaff R, Hernandez-Torres V, Brull SJ, Prielipp RC. Neuromuscular blockade management in the critically III patient. J Intensive Care. 2020 May;8(1):37. https://doi.org/10.1186/ s40560-020-00455-2 PMID:32483489

15. Burtin C, Clerckx B, Robbeets C, Ferdinande P, Langer D, Troosters $T$, et al. Early exercise in critically ill patients enhances shortterm functional recovery. Crit Care Med. 2009 Sep;37(9):2499505. https://doi.org/10.1097/CCM.0b013e3181a38937 PMID:19623052

16. Lightfoot AP, Cooper RG. The role of myokines in muscle health and disease. Curr Opin Rheumatol. 2016 Nov;28(6):6616. https://doi.org/10.1097/BOR.0000000000000337 PMID:27548653

17. Piccirillo R. Exercise-Induced Myokines With Therapeutic Potential for Muscle Wasting. Front Physiol. 2019 Mar;10:287. https://doi. org/10.3389/fphys.2019.00287 PMID:30984014

18. Schreiber A, Bertoni M, Goligher EC. Avoiding Respiratory and Peripheral Muscle Injury During Mechanical Ventilation: Diaphragm-Protective Ventilation and Early Mobilization. Crit Care Clin. 2018 Jul;34(3):357-81. https://doi.org/10.1016/j. ccc.2018.03.005 PMID:29907270

19. Zayed Y, Kheiri B, Barbarawi M, Chahine A, Rashdan L, Chintalapati $S$, et al. Effects of neuromuscular electrical stimulation in critically ill patients: A systematic review and meta-analysis of randomised controlled trials. Aust Crit Care. 2020 Mar;33(2):20310. https://doi.org/10.1016/j.aucc.2019.04.003 PMID:31160215

20. Hultman E, Sjöholm H, Jäderholm-Ek I, Krynicki J. Evaluation of methods for electrical stimulation of human skeletal muscle in situ. Pflugers Arch. 1983 Jul;398(2):139-41. https://doi. org/10.1007/BF00581062 PMID:6622220

21. Sillen MJ, Franssen FM, Gosker HR, Wouters EF, Spruit MA. Metabolic and structural changes in lower-limb skeletal muscle following neuromuscular electrical stimulation: a systematic review. PLoS One. 2013 Sep;8(9):e69391. https://doi.org/10.1371/ journal.pone.0069391 PMID:24019860

22. Maffiuletti NA. Physiological and methodological considerations for the use of neuromuscular electrical stimulation. Eur J Appl Physiol. 2010 Sep;110(2):223-34. https://doi.org/10.1007/ s00421-010-1502-y PMID:20473619

23. Wageck B, Nunes GS, Silva FL, Damasceno MC, de Noronha M. Application and effects of neuromuscular electrical stimulation in critically ill patients: systematic review. Med Intensiva. 2014
Oct;38(7):444-54. https://doi.org/10.1016/j.medin.2013.12.003 PMID:25060511

24. Trethewey SP, Brown N, Gao F, Turner AM. Interventions for the management and prevention of sarcopenia in the critically ill: $A$ systematic review. J Crit Care. 2019 Apr;50:287-95. https://doi. org/10.1016/j.jcrc.2019.01.008 PMID:30673625

25. Gerovasili V, Stefanidis K, Vitzilaios K, Karatzanos E, Politis $\mathrm{P}$, Koroneos $\mathrm{A}$, et al. Electrical muscle stimulation preserves the muscle mass of critically ill patients: a randomized study. Crit Care. 2009;13(5):R161. https://doi.org/10.1186/cc8123 PMID:19814793

26. Rodriguez PO, Setten M, Maskin LP, Bonelli I, Vidomlansky $S R$, Attie $S$, et al. Muscle weakness in septic patients requiring mechanical ventilation: protective effect of transcutaneous neuromuscular electrical stimulation. J Crit Care. 2012 Jun;27(3):319.e1-8. https://doi.org/10.1016/j.jcrc.2011.04.010 PMID:21715139

27. Dirks ML, Hansen D, Van Assche A, Dendale P, Van Loon LJ. Neuromuscular electrical stimulation prevents muscle wasting in critically ill comatose patients. Clin Sci (Lond). 2015 Mar;128(6):35765. https://doi.org/10.1042/CS20140447 PMID:25296344

28. Routsi C, Gerovasili V, Vasileiadis I, Karatzanos E, Pitsolis T, Tripodaki E, et al. Electrical muscle stimulation prevents critical illness polyneuromyopathy: a randomized parallel intervention trial. Crit Care. 2010;14(2):R74. https://doi.org/10.1186/cc8987 PMID:20426834

29. Abu-Khaber HA, Abouelela AM, Abdelkarim EM. Effect of electrical muscle stimulation on prevention of ICU acquired muscle weakness and facilitating weaning from mechanical ventilation. Alex J Med. 2013;49(4):309-15. https://doi.org/10.1016/j. ajme.2013.03.011.

30. Karavidas Al, Raisakis KG, Parissis JT, Tsekoura DK, Adamopoulos $\mathrm{S}$, Korres DA, et al. Functional electrical stimulation improves endothelial function and reduces peripheral immune responses in patients with chronic heart failure. Eur J Cardiovasc Prev Rehabil. 2006 Aug;13(4):592-7. https://doi.org/10.1097/01. hjr.0000219111.02544.ff PMID:16874150

31. Dobšák P, Tomandl J, Spinarova L, Vitovec J, Dusek L, Novakova $M$, et al. Effects of neuromuscular electrical stimulation and aerobic exercise training on arterial stiffness and autonomic functions in patients with chronic heart failure. Artif Organs. 2012 Oct;36(10):920-30. https://doi.org/10.1111/j.15251594.2012.01474.x PMID:22882472

32. Kimura T, Kaneko F, Iwamoto E, Saitoh S, Yamada T. Neuromuscular electrical stimulation increases serum brain-derived neurotrophic factor in humans. Exp Brain Res. 2019 Jan;237(1):47-56. https://doi.org/10.1007/s00221-018-5396-y PMID:30306243

33. Truong AD, Kho ME, Brower RG, Feldman DR, Colantuoni E, Needham DM. Effects of neuromuscular electrical stimulation on cytokines in peripheral blood for healthy participants: a prospective, single-blinded Study. Clin Physiol Funct Imaging 2017 May;37(3):255-62. https://doi.org/10.1111/cpf.12290 PMID:26475418

34. Maffiuletti NA, Roig M, Karatzanos E, Nanas S. Neuromuscular electrical stimulation for preventing skeletal-muscle weakness and wasting in critically ill patients: a systematic review. BMC Med. 2013 May;11(1):137. https://doi.org/10.1186/1741-701511-137 PMID:23701811

35. Parry SM, Berney S, Granger CL, Koopman R, El-Ansary D, Denehy $\mathrm{L}$. Electrical muscle stimulation in the intensive care setting: 
a systematic review. Crit Care Med. 2013 Oct;41(10):240618. https://doi.org/10.1097/CCM.0b013e3182923642 PMID:23921276

36. Burke D, Gorman E, Stokes D, Lennon O. An evaluation of neuromuscular electrical stimulation in critical care using the ICF framework: a systematic review and meta-analysis. Clin Respir J. 2016 Jul;10(4):407-20. https://doi.org/10.1111/crj.12234 PMID:25353646

37. Liu M, Luo J, Zhou J, Zhu X. Intervention effect of neuromuscular electrical stimulation on ICU acquired weakness: A meta-analysis. Int J Nurs Sci. 2020 Mar;7(2):228-37. https://doi.org/10.1016/j. ijnss.2020.03.002 PMID:32685621

38. Gutiérrez-Arias RE, Zapata-Quiroz CC, Prenafeta-Pedemonte BO, Nasar-Lillo NA, Gallardo-Zamorano DI. Effect of Neuromuscular Electrical Stimulation on the Duration of Mechanical Ventilation. Respir Care. 2021 Apr;66(4):679-85. https://doi.org/10.4187/ respcare.08363 PMID:33402382

39. Nussbaum EL, Houghton P, Anthony J, Rennie S, Shay BL, Hoens AM. Neuromuscular Electrical Stimulation for Treatment of Muscle Impairment: Critical Review and Recommendations for Clinical Practice. Physiother Can. 2017;69(5):1-76. https://doi. org/10.3138/ptc.2015-88 PMID:29162949

40. Dos Santos FV, Cipriano G Jr, Vieira L, Güntzel Chiappa AM, Cipriano GB, Vieira P, et al. Neuromuscular electrical stimulation combined with exercise decreases duration of mechanical ventilation in ICU patients: A randomized controlled trial. Physiother Theory Pract. 2020 May;36(5):580-8. https://doi.org/10.1080/09 593985.2018.1490363 PMID:30321084

41. Mayer KP, Thompson Bastin ML, Montgomery-Yates AA, Pastva AM, Dupont-Versteegden EE, Parry SM, et al. Acute skeletal muscle wasting and dysfunction predict physical disability at hospital discharge in patients with critical illness. Crit Care. 2020 Nov;24(1):637. https://doi.org/10.1186/s13054-020-03355-x PMID:33148301

42. Dall' Acqua AM, Sachetti A, Santos LJ, Lemos FA, Bianchi T, Naue WS, et al.; MoVe- ICU Group. Use of neuromuscular electrical stimulation to preserve the thickness of abdominal and chest muscles of critically ill patients: A randomized clinical trial. J Rehabil
Med. 2017 Jan;49(1):40-8. https://doi.org/10.2340/165019772168 PMID:28101565

43. Koutsioumpa E, Makris D, Theochari A, Bagka D, Stathakis S, Manoulakas E, et al. Effect of transcutaneous electrical neuromuscular stimulation on myopathy in intensive care patients. Am J Crit Care. 2018 Nov;27(6):495-503. https://doi.org/10.4037/ ajcc2018311 PMID:30385541

44. Koçan Kurtoglu D, Tastekin N, Birtane M, Tabakoglu E, Sut N. Effectiveness of neuromuscular electrical stimulation on auxiliary respiratory muscles in patients with chronic obstructive pulmonary disease treated in the intensive care unit. Turk Fizik Rehabil Derg. 2015;61(1):12-7. https://doi.org/10.5152/tf$\operatorname{trd} .2015 .04378$.

45. McCaughey EJ, Jonkman AH, Boswell-Ruys $C L$, McBain RA, Bye $E A$, Hudson AL, et al. Abdominal functional electrical stimulation to assist ventilator weaning in critical illness: a doubleblinded, randomised, sham-controlled pilot study. Crit Care. 2019 Jul;23(1):261. https://doi.org/10.1186/s13054-019-2544-0 PMID:31340846

46. Chen YH, Hsiao HF, Li LF, Chen NH, Huang CC. Effects of Electrical Muscle Stimulation in Subjects Undergoing Prolonged Mechanical Ventilation. Respir Care. 2019 Mar;64(3):262-71. https:// doi.org/10.4187/respcare.05921 PMID:30723168

47. Sachetti A, Carpes MF, Dias AS, Sbruzzi G. Safety of neuromuscular electrical stimulation among critically ill patients: systematic review. Rev Bras Ter Intensiva. 2018 Apr-Jun;30(2):219-25. PMID:29995088

48. Lago AF, de Oliveira AS, de Souza HC, da Silva JS, Basile-Filho A, Gastaldi AC. The effects of physical therapy with neuromuscular electrical stimulation in patients with septic shock: study protocol for a randomized cross-over design. Medicine (Baltimore). 2018 Feb;97(6):e9736. https://doi.org/10.1097/ MD.0000000000009736 PMID:29419665

49. Houghton P, Nussbaum EL, Hoens AM. ELECTROPHYSICAL AGENTS - Contraindications And Precautions: An Evidence-Based Approach To Clinical Decision Making In Physical Therapy. Physiother Can. 2010;62(5):1-80. https://doi.org/10.3138/ptc.62.5 PMID:21886384 